University of Nebraska - Lincoln

DigitalCommons@University of Nebraska - Lincoln

Roman L. Hruska U.S. Meat Animal Research

U.S. Department of Agriculture: Agricultural Center

Research Service, Lincoln, Nebraska

2014

\title{
Use of robust multivariate linear mixed models for estimation of genetic parameters for carcass traits in beef cattle
}

\author{
S. O. Peters \\ Berry College, speters@berry.edu \\ lowa State University, Adnan Menderes University \\ D. J. Garrick \\ lowa State University, D.Garrick@massey.ac.nz \\ R. L. Fernando \\ lowa State University, rohan@iastate.edu \\ E. J. Pollak \\ USDA-ARS, jpollak2@unl.edu
}

See next page for additional authors

Follow this and additional works at: https://digitalcommons.unl.edu/hruskareports

Peters, S. O.; lowa State University, Adnan Menderes University; Garrick, D. J.; Fernando, R. L.; Pollak, E. J.; Enns, R. Mark; De Donato, M.; Ajayi, O. O.; and Imumorin, I. G., "Use of robust multivariate linear mixed models for estimation of genetic parameters for carcass traits in beef cattle" (2014). Roman L. Hruska U.S. Meat Animal Research Center. 328.

https://digitalcommons.unl.edu/hruskareports/328

This Article is brought to you for free and open access by the U.S. Department of Agriculture: Agricultural Research Service, Lincoln, Nebraska at DigitalCommons@University of Nebraska - Lincoln. It has been accepted for inclusion in Roman L. Hruska U.S. Meat Animal Research Center by an authorized administrator of DigitalCommons@University of Nebraska - Lincoln. 


\section{Authors}

S. O. Peters; lowa State University, Adnan Menderes University; D. J. Garrick; R. L. Fernando; E. J. Pollak; R. Mark Enns; M. De Donato; O. O. Ajayi; and I. G. Imumorin 
ORIGINAL ARTICLE

\title{
Use of robust multivariate linear mixed models for estimation of genetic parameters for carcass traits in beef cattle
}

\author{
S.O. Peters ${ }^{1}$, K. Kizilkaya ${ }^{2,3}$, D.J. Garrick ${ }^{2}$, R.L. Fernando ${ }^{2}$, E.J. Pollak ${ }^{4}$, R.M. Enns ${ }^{5}$, M. De Donato ${ }^{6,7}$, \\ O.O. Ajayi $^{6}$ \& I.G. Imumorin ${ }^{6}$ \\ 1 Department of Animal Science, Berry College, Mount Berry, GA, USA \\ 2 Department of Animal Science, lowa State University, Ames, IA, USA \\ 3 Department of Animal Science, Adnan Menderes University, Aydin, Turkey \\ 4 Roman L. Hruska US Meat Animal Research Center, USDA-ARS, Clay Center, NE, USA \\ 5 Department of Animal Science, Colorado State University, Fort Collins, CO, USA \\ 6 Animal Genetics and Genomics Laboratory, International Programs, College of Agriculture and Life Sciences, Cornell University, Ithaca, NY, USA \\ 7 IIBCA, Universidad de Oriente, Cumana, Venezuela
}

\section{Keywords}

Carcass growth; genetic parameters; heavy-

tailed distributions; robust models

\section{Correspondence}

S. Peters, Department of Animal Science,

Berry College, Mount Berry, GA 30149, USA.

Tel: (706) 368 6919;

Fax: (706) 2362223 ;

E-mail: speters@berry.edu

Received: 16 July 2013;

accepted: 10 April 2014

\section{Summary}

Assumptions of normality of residuals for carcass evaluation may make inferences vulnerable to the presence of outliers, but heavy-tail densities are viable alternatives to normal distributions and provide robustness against unusual or outlying observations when used to model the densities of residual effects. We compare estimates of genetic parameters by fitting multivariate Normal (MN) or heavy-tail distributions (multivariate Student's $t$ and multivariate Slash, MSt and MS) for residuals in data of hot carcass weight (HCW), longissimus muscle area (REA) and 12th to 13th rib fat (FAT) traits in beef cattle using 2475 records from 2007 to 2008 from a large commercial operation in Nebraska. Model comparisons using deviance information criteria (DIC) favoured MSt over MS and MN models, respectively. The posterior means (and $95 \%$ posterior probability intervals, PPI) of $v$ for the MSt and MS models were $5.89 \pm 0.90(4.35,7.86)$ and $2.04 \pm 0.18(1.70,2.41)$, respectively. Smaller values of posterior densities of $v$ for MSt and MS models confirm that the assumption of normally distributed residuals is not adequate for the analysis of the data set. Posterior mean (PM) and posterior median (PD) estimates of direct genetic variances were variable with MSt having the highest mean value followed by MS and MN, respectively. Posterior inferences on genetic variance were, however, comparable among the models for FAT. Posterior inference on additive heritabilities for HCW, REA and FAT using MN, MSt and MS models indicated similar and moderate heritability comparable with the literature. Posterior means of genetic correlations for carcass traits were variable but positive except for between REA and FAT, which showed an antagonistic relationship. We have demonstrated that genetic evaluation and selection strategies will be sensitive to the assumed model for residuals.

\section{Introduction}

The evaluation of carcass quality and its prerequisite estimation of genetic parameters are of considerable importance in genetic improvement for beef cattle
(Marshall 1994; Crews \& Kemp 2002; Utrera \& Van Vleck 2004; MacNeil et al. 2010). The number of carcass records for most breeds is low when compared to other traits such as birth, weaning, yearling, scrotal circumference and other reproduction data due to 
cost and difficulty of collecting carcass data (Bertrand et al. 2001; Crews \& Kemp 2001, 2002). Like most traits, the data for carcass quality have measurement errors and other types of random non-genetic variation that comprise the residual effects, which are often assumed to be normally distributed with zero mean and common variance (Kizilkaya et al. 2002, 2010; Cardoso et al. 2005, 2007; Kizilkaya \& Tempelman 2005). However, carcass data like most field data exhibit more outliers than would be expected if the distributions of residual effects really were normally distributed. Heavy-tailed densities have been reported to be viable alternatives to assuming Normal distributions and provide robustness against unusual or outlying observations when used to model the densities of residual effects (Kizilkaya et al. 2003, 2010; Kizilkaya \& Tempelman 2005; Peters et al. 2013). Among a number of alternative distributions that exhibit heavy tails, Student's $t$ and Slash distributions are appealing because they are symmetric and converge to Normal distributions as their corresponding degrees of freedom exceed 50 and 20, respectively (Peters et al. 2013). Many authors have advocated the use of multivariate approaches to genetic evaluation not only because it is more accurate and precise but also because even moderately inheritable traits can benefit from information from correlated traits (Stranden \& Gianola 1999; Kizilkaya et al. 2003, 2010; Kizilkaya \& Tempelman 2005). Genetic parameter estimation for carcass traits has been reported to be hampered by the small number of records available (Meyer 2007). Multivariate approaches that directly model all measurements on one trait category, such as carcass traits, by estimating covariance structure may better utilize the information in the data set and provide trait-specific estimates of genetic effects at the cost of greater computational burden and more complex interpretation of the results (Meyer 2007; Peters et al. 2013). Meyer (2007) published multivariate restricted maximum likelihood estimates of genetic (co)variances for carcass traits of Angus cattle, by fitting a number of reduced rank and factor analytic models. There are reports of application of heavy-tailed distributions to growth and calving difficulty data in literature (Kizilkaya et al. 2003; Peters et al. 2013), but there are no reports of application of heavy-tailed distribution to carcass data. In this study, we compared results assuming multivariate Normal distributions to those fitting multivariate Student's $t$ (MSt) or multivariate Slash (MS) distributions with unknown degrees of freedom for the residuals in multivariate carcass data.

\section{Materials and methods}

Data set

Carcass records for this study came from a large commercial ranch with a composite cattle population of approximately 20000 animals located in the sand hills of Nebraska. A total of 2475 hot carcass weight (HCW), longissimus muscle area (REA) and fat thickness (FAT) records were obtained from 2007 to 2008. The information contained in the data set included pedigree information, contemporary groups, feedlot pen and gender. The pedigree of 7616 individuals was used to define additive genetic effects. Contemporary groups with 18 levels created by combining years and pastures were used as a fixed effect in the analysis (Peters et al. 2013). In addition, the factors of feedlot pen with 11 levels and gender with bull, steer, heifers and cow levels also were considered as other fixed effects in the analysis. A summary of data set across contemporary group, feedlot pen and gender effects is shown in Table 1 .

\section{Statistical analysis}

Multiple trait analysis of HCW, REA and FAT was carried out using a scale-mixture MN model (Rosa et al. 2003) which is defined for animal $i$ as follows:

$$
\mathbf{y}_{i}=\mathbf{X}_{i} \mathbf{b}+\mathbf{Z}_{i} \mathbf{a}+\frac{\mathbf{e}_{i}}{\sqrt{\lambda_{i}}},
$$

where $\mathbf{y}_{i}=\left[y_{i, \mathrm{HCW}}, y_{i, \mathrm{REA}}, y_{i, \mathrm{FAT}}\right]^{\prime}$ is the vector of phenotypic values of HCW, REA and FAT for animal $i$; $\mathbf{b}$ includes fixed effects of contemporary group, feedlot pen and gender; $\mathbf{a}$ is a vector of random genetic animal effects for HCW, REA and FAT for 7616 animals in the pedigree. $\mathbf{X}_{i}$ and $\mathbf{Z}_{i}$ are the corresponding incidence matrices for fixed and random genetic effects. The residuals $\mathbf{e}_{i}=\left[e_{i, \mathrm{HCW}}, e_{i}\right.$ REA, $\left.e_{i, \mathrm{FAT}}\right]^{\prime}$ were assumed to follow a $\mathrm{MN}$ distribution with null means and (co)variance matrix $\mathbf{R}_{0}$, where

$$
\mathbf{R}_{0}=\left[\begin{array}{ccc}
\sigma_{e_{\mathrm{HCW}}}^{2} & \sigma_{e_{\mathrm{HCW}, \mathrm{REA}}} & \sigma_{e_{\mathrm{HCW}, \mathrm{FAT}}} \\
\sigma_{e_{\mathrm{REA}, \mathrm{HCW}}} & \sigma_{e_{\mathrm{REA}}}^{2} & \sigma_{e_{\mathrm{REA}, \mathrm{FAT}}} \\
\sigma_{e_{\mathrm{FAT}, \mathrm{HCW}}} & \sigma_{e_{\mathrm{FAT}, \mathrm{REA}}} & \sigma_{e_{\mathrm{FAT}}}^{2}
\end{array}\right] .
$$

The scalar $\lambda_{i}$ is an unknown positive random variable for animal $i$, independent of $\mathbf{e}_{i}$.

A flat prior was assumed for the fixed effects (b). Genetic effects (a) were assumed to be distributed as MN with null mean vector (0) and (co)variance matrix $\mathbf{G}_{0} \otimes \mathbf{A}$, where $\mathbf{A}$ is the numerator relationship matrix, $\mathbf{G}_{0}$ is the (co)variance matrix 
Table 1 Summary statistics for hot carcass weight (HCW), longissimus muscle area (REA) and fat thickness (FAT) obtained from beef cattle in Rex Ranch across gender, contemporary group and feedlot pen effects

\begin{tabular}{|c|c|c|c|c|}
\hline Effects & $\mathrm{n}$ & HCW (kg) & $\operatorname{REA}\left(\mathrm{cm}^{2}\right)$ & FAT $(\mathrm{cm})$ \\
\hline \multicolumn{5}{|l|}{ Gender } \\
\hline Bull & 1227 & $352.7 \pm 32.6$ & $81.6 \pm 8.4$ & $1.31 \pm 0.41$ \\
\hline Steer & 1209 & $356.7 \pm 32.6$ & $82.8 \pm 8.2$ & $1.30 \pm 0.42$ \\
\hline Cow & 23 & $358.9 \pm 32.3$ & $83.1 \pm 11.1$ & $1.33 \pm 0.44$ \\
\hline Heifer & 16 & $344.3 \pm 36.3$ & $79.9 \pm 6.3$ & $1.21 \pm 0.37$ \\
\hline \multicolumn{5}{|c|}{ Contemporary group } \\
\hline 2007_1 & 231 & $351.4 \pm 37.8$ & $83.5 \pm 8.8$ & $1.19 \pm 0.42$ \\
\hline 2007_2 & 197 & $358.0 \pm 32.6$ & $83.1 \pm 9.0$ & $1.42 \pm 0.42$ \\
\hline 2007_3 & 144 & $361.0 \pm 33.9$ & $84.6 \pm 8.0$ & $1.30 \pm 0.40$ \\
\hline 2007_4 & 91 & $369.8 \pm 26.9$ & $84.3 \pm 9.4$ & $1.33 \pm 0.36$ \\
\hline 2007_5 & 66 & $365.1 \pm 29.4$ & $84.1 \pm 9.3$ & $1.32 \pm 0.43$ \\
\hline $2007 \_6$ & 337 & $362.4 \pm 30.8$ & $83.9 \pm 9.1$ & $1.28 \pm 0.40$ \\
\hline 2007_7 & 153 & $344.0 \pm 31.1$ & $82.7 \pm 9.5$ & $1.27 \pm 0.43$ \\
\hline 2007_8 & 14 & $342.3 \pm 35.1$ & $78.6 \pm 11.6$ & $1.34 \pm 0.49$ \\
\hline 2008_1 & 232 & $351.5 \pm 30.3$ & $81.1 \pm 7.3$ & $1.38 \pm 0.41$ \\
\hline 2008_2 & 176 & $345.7 \pm 30.2$ & $79.3 \pm 7.5$ & $1.30 \pm 0.41$ \\
\hline 2008_3 & 115 & $354.1 \pm 30.7$ & $78.6 \pm 6.5$ & $1.44 \pm 0.42$ \\
\hline $2008 \_4$ & 27 & $328.2 \pm 38.1$ & $79.4 \pm 7.3$ & $1.06 \pm 0.42$ \\
\hline 2008_9 & 28 & $342.5 \pm 25.7$ & $79.3 \pm 5.8$ & $1.22 \pm 0.38$ \\
\hline 2008_5 & 112 & $341.0 \pm 28.6$ & $80.3 \pm 7.7$ & $1.19 \pm 0.38$ \\
\hline $2008 \_6$ & 386 & $357.2 \pm 31.0$ & $82.9 \pm 7.1$ & $1.32 \pm 0.43$ \\
\hline 2008_10 & 68 & $349.0 \pm 32.1$ & $79.2 \pm 6.1$ & $1.22 \pm 0.37$ \\
\hline 2008_11 & 71 & $354.3 \pm 37.4$ & $79.4 \pm 7.2$ & $1.33 \pm 0.34$ \\
\hline 2008_8 & 27 & $351.5 \pm 36.2$ & $81.6 \pm 8.0$ & $1.30 \pm 0.42$ \\
\hline \multicolumn{5}{|c|}{ Feedlot pen } \\
\hline 1 & 235 & $349.3 \pm 34.1$ & $83.1 \pm 9.5$ & $1.27 \pm 0.44$ \\
\hline 2 & 255 & $362.0 \pm 27.5$ & $83.3 \pm 9.0$ & $1.33 \pm 0.40$ \\
\hline 3 & 171 & $367.9 \pm 30.4$ & $84.8 \pm 9.5$ & $1.25 \pm 0.37$ \\
\hline 4 & 255 & $361.9 \pm 31.4$ & $83.6 \pm 8.7$ & $1.43 \pm 0.41$ \\
\hline 5 & 244 & $346.4 \pm 39.0$ & $82.9 \pm 8.3$ & $1.17 \pm 0.41$ \\
\hline 6 & 73 & $368.4 \pm 25.9$ & $86.5 \pm 9.4$ & $1.31 \pm 0.39$ \\
\hline 7 & 194 & $350.3 \pm 33.9$ & $79.7 \pm 6.8$ & $1.27 \pm 0.37$ \\
\hline 8 & 268 & $353.9 \pm 31.0$ & $80.7 \pm 7.1$ & $1.47 \pm 0.43$ \\
\hline 9 & 256 & $349.1 \pm 30.2$ & $79.1 \pm 7.3$ & $1.25 \pm 0.36$ \\
\hline 10 & 263 & $348.5 \pm 31.4$ & $81.5 \pm 7.3$ & $1.27 \pm 0.42$ \\
\hline 11 & 261 & $356.2 \pm 32.1$ & $82.8 \pm 7.4$ & $1.29 \pm 0.42$ \\
\hline Overall & 2475 & $354.7 \pm 32.7$ & $82.2 \pm 8.3$ & $1.30 \pm 0.41$ \\
\hline
\end{tabular}

$$
\mathbf{G}_{0}=\left[\begin{array}{ccc}
\sigma_{\mathrm{a}_{\mathrm{HCW}}}^{2} & \sigma_{\mathrm{a}_{\mathrm{H} C W, \mathrm{REA}}} & \sigma_{\mathrm{a}_{\mathrm{HCW}, \mathrm{FAT}}} \\
\sigma_{\mathrm{a}_{\mathrm{REA}, \mathrm{HCW}}} & \sigma_{\mathrm{a}_{\mathrm{REA}}}^{2} & \sigma_{\mathrm{a}_{\mathrm{REA}, \mathrm{FAT}}} \\
\sigma_{\mathrm{a}_{\mathrm{FAT}, \mathrm{HCW}}} & \sigma_{\mathrm{a}_{\mathrm{FAT}, \mathrm{REA}}} & \sigma_{\mathrm{a}_{\mathrm{FAT}}}^{2}
\end{array}\right]
$$

and $\otimes$ denotes the Kronecker product.

Covariance matrices $\mathbf{G}_{0}$ and $\mathbf{R}_{0}$ are assumed to follow, a priori, a scaled three-dimensional inverse Wishart distributions $I W\left(\mathbf{V}_{G}, v_{G}\right)$ and $I W\left(\mathbf{V}_{R}, v_{R}\right)$ with $\mathbf{V}_{G}$, $\mathbf{V}_{R}$ and $v_{G}, v_{R}$ parameters, respectively. However, flat prior distributions were assigned to $\mathbf{G}_{0}$ and $\mathbf{R}_{0}$ by setting $\mathbf{V}_{G}=\mathbf{V}_{R}=0$ and $v_{G}=v_{R}=-4$ (Sorensen $\&$ Gianola 2002).

In the MN model, there is no distributional specification for $\lambda_{i}$ in equation (1), because 1 assigned to $\lambda_{i}$ for all $i=1,2, \ldots, n$. In the MSt model with $v_{\mathrm{MS} t}$ degrees of freedom, the distribution of $\lambda_{i}$ in equation (1) is a $\operatorname{Gamma}\left(v_{\mathrm{MSt}} / 2, v_{\mathrm{MSt}} / 2\right)$ distribution where $v_{\mathrm{MSt}}>0$. A prior of $\mathrm{p}\left(v_{\mathrm{MS} t}\right)=1 /\left(1+v_{\mathrm{MS} t}\right)^{2}$ for $v_{\text {MSt }}>2$ was assigned to $v_{\text {MSt }}$ (Kizilkaya et al. 2003). In the MS model, the distribution of $\lambda_{i}$ in equation (1) is a $\operatorname{Beta}(v, 1)$ distribution with density function $p\left(\lambda_{i} \mid v_{\mathrm{MS}}\right)=v_{\mathrm{MS}} \lambda_{i}^{v_{\mathrm{MS}}-1}$ where $0<\lambda_{i} \leq 1$, and $v_{\mathrm{MS}}$ is the degrees of freedom parameter. A truncated conjugate Gamma prior $(\alpha, \beta)$ was assigned to $v_{M S}$, in which the shape parameter $\alpha$ is equal to 0.015 and the inverse scale parameter $\beta$ is equal to 0.001 , so that the prior density is then $p\left(v_{\mathrm{MS}} \mid \alpha, \beta\right) \propto v_{\mathrm{MS}}^{\alpha-1} \exp \left\{-\beta v_{\mathrm{MS}}\right\}$ $I\left\{v_{\text {MS }}>1\right\}$ with $v_{\text {MS }}>1$ (Kizilkaya et al. 2003, 2010).

Inferences on parameters of interest in MSt and MS models can be made from the conditional posterior distributions using Markov chain Monte Carlo (MCMC) methods. The fully conditional posterior distributions of each of the unknown parameters are used to generate proposal samples from the target distribution. The fully conditional posterior distributions of fixed (b) and genetic (a) effects are MN with mean $[\hat{\mathbf{b}}, \hat{\mathbf{a}}]$ and covariance matrix $\mathbf{C}$, where $[\hat{\mathbf{b}}, \hat{\mathbf{a}}]$ are solutions to Henderson's mixed model equations constructed with heterogeneous residual variances $\mathbf{R}_{0} \lambda_{i}^{-1}$, and $C$ is the inverse of this mixed model coefficient matrix (Stranden \& Gianola 1999; Kizilkaya et al. 2010). The (co)variance matrices $\mathbf{G}_{0}$ and $\mathbf{R}_{0}$ have inverse Wishart conditional posterior distributions, which are constructed from $[\hat{\mathbf{b}}, \hat{\mathbf{a}}, \hat{\lambda}]$ where $\hat{\lambda}$ is solution for $\lambda_{i}$ (Sorensen $\&$ Gianola 2002).

The fully conditional posterior of $\lambda_{i}$ for the MSt model is a Gamma distribution with shape $\left(v_{\mathrm{MS} t}+3\right) /$ 2 and scale $0.5\left[\mathbf{e}_{i} \mathbf{R}_{0}^{-1} \mathbf{e}_{i}+v_{\mathrm{MSt}}\right]$. The fully conditional posterior distribution of $v_{\mathrm{MS} t}$ for the MSt model does not have a standard form, and a random-walk Metropolis-Hasting ( $\mathrm{MH}$ ) algorithm (Chib \& Greenberg 1995) was used to draw samples for $v_{\text {MSt }}$ (Kizilkaya et al. 2003, 2010).

The fully conditional posterior distribution of $\lambda_{i}$ for MS model is a Truncated-Gamma distribution for $0<\lambda_{i}<1$, with shape $v_{\mathrm{MS}}+3 / 2$ and scale 0.5 $\left[\mathbf{e}_{i} \mathbf{R}_{0}^{-1} \mathbf{e}_{i}\right]$. The fully conditional posterior distribution of $v_{\mathrm{MS}}$ is a Gamma distribution $\operatorname{Gamma}(n+\alpha$, $\beta-\sum_{i=1}^{n} \log \lambda_{i}$ ) with $v_{\mathrm{MS}}>1$ (Rosa et al. 2003).

Marginal residual variances, heritabilities and genetic correlations for multivariate Normal, Student's $t$ and Slash models

The marginal residual (co)variance parameters $\left(\mathbf{R}_{E}\right)$ for MN, MSt and MS models were calculated using 
$\mathbf{R}_{E}=\mathbf{R}_{0}, \quad \mathbf{R}_{E}=\mathbf{R}_{0}\left(v_{\mathrm{MS} t}\right) /\left(v_{\mathrm{MS} t}-2\right)$ where $v_{\mathrm{MS} t}>2$ and $\mathbf{R}_{E}=\mathbf{R}_{0}\left(v_{\mathrm{MS}}\right) /\left(v_{\mathrm{MS}}-1\right)$ where $v_{\mathrm{MS}}>1$, respectively, as given by Stranden and Gianola (1999) and Cardoso et al. (2005).

Direct $\left(h_{\mathrm{a}_{i, k}}^{2}\right)$ heritability and genetic correlation $\left(r_{j, j^{\prime}, k}\right)$ estimates were obtained from estimates of (co)variance components based on: $h_{\mathrm{a}_{j, k}}^{2}=\left(\sigma_{\mathrm{a}_{j, k}}^{2}\right) /\left(\sigma_{\mathrm{a}_{j, k}}^{2}+\sigma_{E_{j, k}}^{2}\right)$ and $r_{j, j^{\prime}, k}=\left(\sigma_{\mathrm{a}_{j, j^{\prime}, k}}\right) /\left(\sqrt{\sigma_{\mathrm{a}_{j, k}}^{2} \sigma_{\mathrm{a}_{j^{\prime}, k}}^{2}}\right)$ where $j$ and $j \neq j^{\prime}$ represent traits HCW, REA or FAT, and $k$ represents the model for residuals being either MN, MSt or MS (Kizilkaya et al. 2010; Peters et al. 2013).

\section{Markov chain Monte Carlo implementation and model comparison}

Three separate MCMC chains of 360000 cycles were generated using different starting values for each of the MN, MSt or MS models. Graphical inspection (time series traces) of the iterations were used to determine a burn-in period of 10000 defined as the number of iterations discarded at the start of the MCMC chain to ensure sampling from the correct marginal distributions. A further 350000 post-burnin MCMC cycles were generated for each of the MN, MSt or MS models. Every successive post-burn-in sample was retained, so that 350000 samples were used to infer posterior distributions of unknown parameters. Posterior means of the parameters were obtained from their respective marginal posterior densities. Interval estimates were determined as posterior probability intervals (PPI) obtained from the 2.5 and 97.5 percentiles of each posterior density to provide 95\% PPI.

The effective sample size (ESS) of the samples (Sorensen et al. 1995) was used to determine the effectiveness of MCMC mixing after burn-in period. The ESS is an estimate on the information content of the MCMC samples in terms of an equivalent number of independent samples.

Model comparisons were carried out using the deviance information criteria (DIC) proposed by Spiegelhalter et al. (2002) for alternative constructions of hierarchical models. The DIC is based on the posterior distribution of the deviance statistic, which is -2 times the sampling distribution of the data as specified in the first stage of a hierarchical model. The calculation of DIC was carried out as the sum of average Bayesian deviance $(\bar{D})$ plus the effective number of parameters $\left(p_{D}\right)$ with respect to the models MSt, MS or MN. A smaller DIC value was used as an indicator of better fit to the multivariate data set (Peters et al. 2013).

\section{Results and discussion}

The overall mean value of $354.7 \mathrm{~kg}$ for HCW recorded in this study (Table 1) is higher than values reported by Riley et al. (2002) for Brahmans; Crews et al. (2003) for Simmental and Meyer (2007) for Australian Angus. This may be due to the fact the animals evaluated are composites or a result of breed differences. Marshall (1994) reported that carcass composition tends not to be improved by heterosis, but he went further to conclude that heterotic effects on carcass weight at a given age are positive.

The overall mean value of $82.2 \mathrm{~cm}^{2}$ for REA presented in Table 1 is lower than those reported for Charolais, Gelbvieh and Limousin in Marshall (1994). They are also $10 \mathrm{~cm}^{2}$ lower than values reported by Riley et al. (2002) for Brahmans raised in Florida. Nephawe et al. (2004) also reported a slightly lower value from the data of the Germplasm Evaluation Program (GPE) at the U.S. Meat Animal Research Center (USMARC), Clay Center, NE. The differences herein are likely the result of the bull selection programme of the commercial ranch and of breed differences. For example, this population contains no Bos indicus influenced animals.

The overall mean value of $1.30 \mathrm{~cm}$ for FAT reported in this study is higher than values reported by Peters et al. (2012) for ultrasound measure of FAT in Brangus females but comparable to values reported by Nephawe et al. (2004)

Based on the raw trace plots of samples of 360000 iterations, it was determined that 10000 iterations were sufficiently long burn-in period for all variancecovariance components, degrees of freedom parameters and within all chains. The length of the burn-in period was enough to eliminate the effect of the different starting values.

Table 2 shows the estimated ESS for each variancecovariance component and degrees of freedom. They are based on a sum of separate determinations from each of the three separate chains. The ESS in Table 2 was from 302 to 6490, pointing out sufficient MCMC mixing for parameters. The ESS of 100 was suggested as the minimum ESS for reliable statistical inference (Bink et al. 1998). The ESS for variance-covariances from robust model (MSt and MS) was found to be higher than those from normal model $(\mathrm{MN})$, indicating better mixing in the robust models.

Multivariate analyses of carcass traits produced DIC values of 41 998, 40621 and 41058 for MN, MSt and MS, respectively. Kizilkaya et al. (2002, 2003) reported that MSt was the best-fitting model based on smaller DIC values. This result was consistent with 
Table 2 Effective sample sizes (ESS) for additive genetic variance-covariances $\left(\sigma_{a}^{2}\right)$ using multivariate Normal (MN), Student's $t$ (MSt) or Slash (MS) models for hot carcass weight (HCW), longissimus muscle area (REA) and fat thickness (FAT)

\begin{tabular}{lrrr}
\hline Parameter & MN & MSt & MS \\
\hline$\sigma_{a_{\text {HCW }}}^{2}$ & 374 & 1264 & 781 \\
$\sigma_{a_{\text {REA }}}^{2}$ & 1112 & 2358 & 1788 \\
$\sigma_{a_{\text {FAT }}}^{2}$ & 681 & 919 & 807 \\
$\sigma_{\text {HCW,REA }}$ & 425 & 2417 & 1332 \\
$\sigma_{\text {HCW,FAT }}$ & 302 & 1452 & 842 \\
$\sigma_{\text {REA,FAT }}$ & 673 & 2417 & 1529 \\
$V$ & - & 2541 & 6490 \\
\hline
\end{tabular}

previous studies that showed that Student's $t$ models were better fit to clinical mastitis (Chang et al. 2006), postweaning gain (Cardoso et al. 2007), birthweight and gestation length traits when compared to slash or Normal distributions (Kizilkaya et al. 2010). Cardoso et al. (2005) demonstrated that normally distributed residuals are not the best fit for growth data. They looked at three alternative specifications for the marginal density of residuals (i.e. Normal, Student's $t$ or Slash). In their study, pseudo-Bayes factor derived from log marginal likelihood function showed that a heteroskedastic t-error model provided the best fit to the data among the six different residual specifications considered. They concluded that the conventional homoskedastic Gaussian error model in which residuals were assumed Normal, independently and identically distributed with common residual variance, was the poorest choice for the data set. To our knowledge, this is the first comparison and application of heavytailed distribution to carcass data in beef cattle.

The posterior distributions of $v$ from MSt and MS models are in Figure 1. The posterior means of $v$ for
MSt and MS models were 5.89 and 2.04, respectively, with $95 \%$ of PPI of $4.35-7.86$ and $1.70-2.41$ (Table 3). These densities characterized by small values of $v$ for MSt and MS models, which are less than 50 and 20 further supporting that the assumption of normally distributed residuals is inadequate for analysis of HCW, REA and FAT data sets. These results are similar to previous reports of Albert and Chibs (1993), Cardoso et al. (2005); Kizilkaya et al. (2002, 2010). All these authors found that a low value for the degrees of freedom parameter suggesting that the underlying distribution was not normal.

Posterior mean and 95\% PPI estimates of $\sigma_{a}^{2}$ genetic variances from MN, MSt and MS models for HCW, REA and FAT are in Table 4. PM estimate of $\sigma_{a}^{2}$ for HCW was highest $(337.48 \pm 85.18)$ for MSt, followed by MS $(256.14 \pm 87.69)$ with the least value (156.60 \pm 92.39 ) reported for MN. PM estimate of $\sigma_{a}^{2}$ for REA was highest $(34.31 \pm 5.71)$ in MSt followed by MS model, while the lowest value was produced by MN model (Table 4 ). The PM estimates of $\sigma_{a}^{2}$ for FAT were $0.04 \pm 0.02,0.04 \pm 0.01$ and $0.03 \pm 0.01$ for MN, MSt and MS, which were comparable for the three models. The $95 \%$ PPI estimates of $\sigma_{a}^{2}$ from MN, MSt and MS models for HCW, REA or FAT overlapped indicating non-significant difference between PM estimates of those models for HCW, REA or FAT.

The PM of genetic covariances between additive effects is reported in Table 5. The 95\% PPI for covariance between HCW and REA, and covariance between HCW and FAT included zero for MN, MSt and MS models indicating that the correlation between HCW and REA, and between HCW and FAT is non-significant. The $95 \%$ PPI for covariance between REA and FAT did not include zero for MSt and MS models indicating significant antagonistic
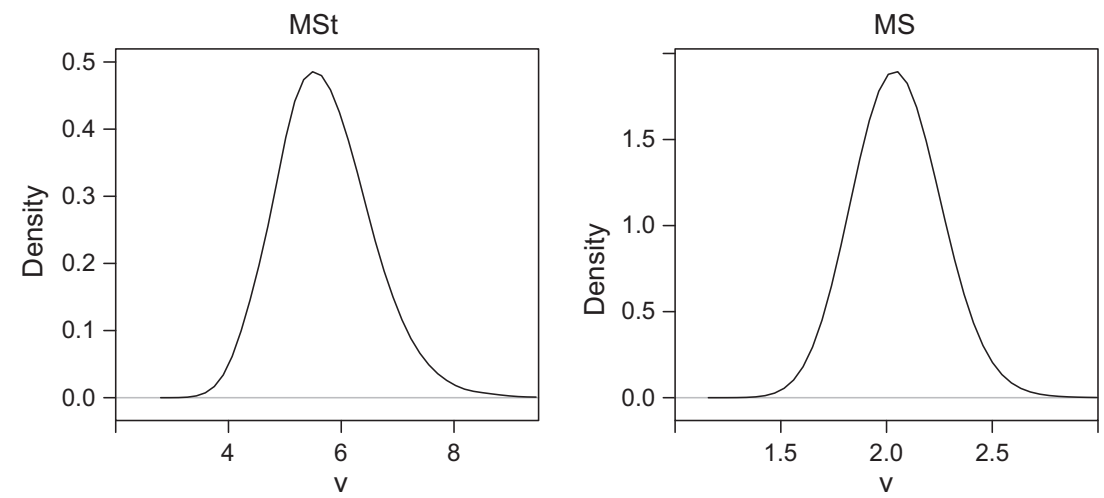

Figure 1 Posterior densities of degrees of freedom (v) obtained from multivariate Student's $t$ (MSt) and Slash (MS) models fitted to hot carcass weight (HCW), longissimus muscle area (REA) and fat thickness (FAT). 
Table 3 Posterior inference for degrees of freedom ( $v$ ) for hot carcass weight (HCW), longissimus muscle area (REA) and fat thickness (FAT) using Student's $t$ (MSt) and Slash (MS) models

\begin{tabular}{|c|c|c|c|c|c|c|}
\hline \multirow[b]{2}{*}{ Degrees of freedom } & \multicolumn{3}{|l|}{ MSt } & \multicolumn{3}{|l|}{ MS } \\
\hline & $\mathrm{PM} \pm \mathrm{SD}$ & PD & $95 \% \mathrm{PPI}$ & $\mathrm{PM} \pm \mathrm{SD}$ & PD & $95 \% \mathrm{PPI}$ \\
\hline$v$ & $5.89 \pm 0.90$ & 5.82 & $4.35 ; 7.86$ & $2.04 \pm 0.18$ & 2.03 & $1.70 ; 2.41$ \\
\hline
\end{tabular}

PM, posterior mean; PD, posterior median; 95\% PPI, 95\% posterior probability interval; SD, posterior standard deviation.

relationship between REA and FAT. The covariance was, however, non-significant for FAT using the MN model (Table 5).

Posterior densities of $h_{a}^{2}$ estimates from MN, MSt and MS models for HCW, REA and FAT are in Figure 2. PM estimates of $0.34 \pm 0.08$ and $0.25 \pm 0.09$ for MSt and MS were higher than $0.16 \pm 0.09$ for MN in HCW (Table 6). These mean values were higher than those reported by Crews et al. (2003). It is, however, important to note that the $h_{a}^{2}$ from MSt in this study were within the range of $h_{a}^{2}$ (0.31-0.64) provided by Marshall (1994) for a population that included Angus, Hereford, Simmental and many unidentified cross-breds and would therefore be of similar breed type as the composites in this study. Utrera and Van Vleck (2004) reported a mean heritability value of 0.40 from meta-analyses of 72 papers published between 1962 and 2004. They noted that heritability for HCW varied widely in the literature, and it could be due to breed group, method of estimation, number of records, types of effect included in the model, sex and measurement errors. For REA, the posterior mean $(0.51 \pm 0.09)$ of $h_{a}^{2}$ for MSt was the highest, followed by MS $(0.45 \pm 0.09)$ and then MN (0.43 \pm 0.12$)$ models (Table 6). The mean values from the three models compared seemed different; however, the respective 95\% PPI for MN, MSt and MS models did overlap. The values reported in this study are higher than ultrasound measure of REA reported by Peters et al. (2012) but within the range reported in literature (Riley et al. 2002; Crews et al. 2003; Utrera \& Van Vleck 2004). The moderate mean values of heritability for REA from this population further confirm that this important carcass trait will respond to selection. For FAT, the PM estimate of $h_{a}^{2}$ from MN (0.24 \pm 0.09$)$ and MSt $(0.24 \pm 0.08)$ models was found to be same; however, MSt model resulted in narrower $95 \%$ PPI $(0.10 ; 0.39)$ of $h_{a}^{2}$ than that $(0.09 ; 0.45)$ from $M N$ model. The smaller value $(0.20 \pm 0.07)$ was found when the data were modelled with MS. The heritability value reported for FAT in this study is lower than many values reported in literature (Marshall 1994; Peters et al. 2012) but low heritability estimates for FAT have also been reported

Table 4 Posterior inference of additive genetic variance $\left(\sigma_{a}^{2}\right)$ for hot carcass weight (HCW), longissimus muscle area (REA) and fat thickness (FAT) using multivariate Normal (MN), Student's $t$ (MSt) or Slash (MS) models

\begin{tabular}{|c|c|c|c|c|c|c|c|c|c|}
\hline \multirow{2}{*}{$\begin{array}{l}\text { Genetic } \\
\text { variance }\end{array}$} & \multicolumn{3}{|l|}{$\mathrm{MN}$} & \multicolumn{3}{|l|}{ MSt } & \multicolumn{3}{|l|}{ MS } \\
\hline & $\mathrm{PM} \pm \mathrm{SD}$ & PD & $95 \% \mathrm{PPI}$ & $\mathrm{PM} \pm \mathrm{SD}$ & PD & $95 \% \mathrm{PPI}$ & $\mathrm{PM} \pm \mathrm{SD}$ & PD & $95 \% \mathrm{PPI}$ \\
\hline$\sigma_{a_{\mathrm{HCW}}}^{2}$ & $156.60 \pm 92.39$ & 131.14 & $43.99 ; 384.08$ & $337.48 \pm 85.18$ & 338.69 & $172.35 ; 502.15$ & $256.14 \pm 87.69$ & 255.13 & $95.44 ; 429.30$ \\
\hline$\sigma_{a_{R E A}}^{2}$ & $28.56 \pm 7.80$ & 28.29 & $14.22 ; 44.31$ & $34.31 \pm 5.71$ & 34.35 & $23.00 ; 45.37$ & $30.85 \pm 6.09$ & 30.64 & $19.47 ; 42.97$ \\
\hline$\sigma_{a_{F A T}}^{2}$ & $0.04 \pm 0.02$ & 0.04 & $0.01 ; 0.08$ & $0.04 \pm 0.01$ & 0.04 & $0.02 ; 0.07$ & $0.03 \pm 0.01$ & 0.03 & $0.01 ; 0.06$ \\
\hline
\end{tabular}

PM, posterior mean; PD, posterior median; 95\% PPI, 95\% posterior probability interval; SD, posterior standard deviation.

Table 5 Posterior inference on additive (a) genetic covariances for hot carcass weight (HCW), longissimus muscle area (REA) and fat thickness (FAT) using multivariate Normal (MN), Student's $t$ (MSt) and Slash (MS) models

\begin{tabular}{|c|c|c|c|c|c|c|c|c|c|}
\hline \multirow[b]{2}{*}{ Genetic covariance } & \multicolumn{3}{|l|}{$\mathrm{MN}$} & \multicolumn{3}{|l|}{ MSt } & \multicolumn{3}{|l|}{ MS } \\
\hline & $\mathrm{PM} \pm \mathrm{SD}$ & PD & $95 \% \mathrm{PPI}$ & $\mathrm{PM} \pm \mathrm{SD}$ & PD & $95 \% \mathrm{PPI}$ & $\mathrm{PM} \pm \mathrm{SD}$ & PD & $95 \% \mathrm{PPI}$ \\
\hline$\sigma_{\mathrm{HCW}, \mathrm{REA}}$ & $20.42 \pm 23.00$ & 19.50 & $-22.19 ; 65.34$ & $26.63 \pm 14.19$ & 26.70 & $-1.13 ; 54.41$ & $15.24 \pm 15.67$ & 15.44 & $-14.88 ; 45.99$ \\
\hline$\sigma_{\mathrm{HCW}, \mathrm{FAT}}$ & $0.06 \pm 1.07$ & 0.03 & $-1.79 ; 2.20$ & $0.55 \pm 0.66$ & 0.56 & $-0.76 ; 1.83$ & $0.15 \pm 0.66$ & 0.13 & $-1.12 ; 1.48$ \\
\hline$\sigma_{\text {REA,FAT }}$ & $-0.41 \pm 0.30$ & -0.41 & $-0.98 ; 0.18$ & $-0.53 \pm 0.16$ & -0.53 & $-0.85 ;-0.21$ & $0.56 \pm 0.18$ & -0.56 & $-0.90 ;-0.19$ \\
\hline
\end{tabular}

PM, posterior mean; PD, posterior median; 95\% PPI, 95\% posterior probability interval; SD, posterior standard deviation. 

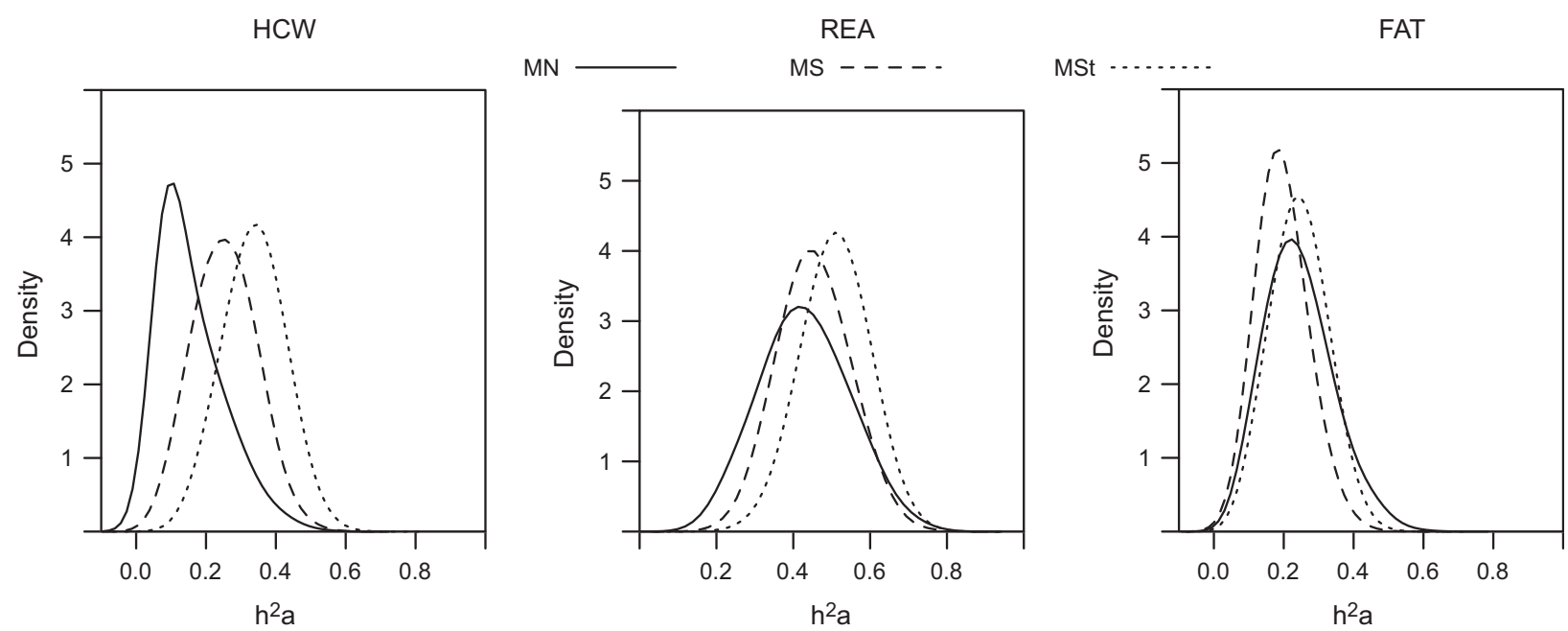

Figure 2 Posterior densities of $\left(h_{a}^{2}\right)$ heritabilities of hot carcass weight (HCW), longissimus muscle area (REA) and fat thickness (FAT) obtained from multivariate Normal (MN), Student's $t$ (MSt) and Slash (MS) models.

Table 6 Posterior inference for heritabilities $\left(h_{a}^{2}\right)$ for hot carcass weight (HCW), longissimus muscle area (REA) and fat thickness (FAT) using multivariate Normal (MN), Student's $t$ (MSt) and Slash (MS) models

\begin{tabular}{|c|c|c|c|c|c|c|c|c|c|}
\hline \multirow[b]{2}{*}{ Heritability } & \multicolumn{3}{|l|}{ MN } & \multicolumn{3}{|l|}{ MSt } & \multicolumn{3}{|l|}{ MS } \\
\hline & $\mathrm{PM} \pm \mathrm{SD}$ & PD & 95\%PPI & $\mathrm{PM} \pm \mathrm{SD}$ & PD & 95\%PPI & $\mathrm{PM} \pm \mathrm{SD}$ & PD & 95\%PPI \\
\hline$h_{a_{\mathrm{HCW}}}^{2}$ & $0.16 \pm 0.09$ & 0.13 & $0.04 ; 0.38$ & $0.34 \pm 0.08$ & 0.34 & $0.17 ; 0.50$ & $0.25 \pm 0.09$ & 0.25 & $0.09 ; 0.43$ \\
\hline$h_{a_{R E A}}^{2}$ & $0.43 \pm 0.12$ & 0.43 & $0.22 ; 0.66$ & $0.51 \pm 0.09$ & 0.51 & $0.34 ; 0.67$ & $0.45 \pm 0.09$ & 0.45 & $0.29 ; 0.63$ \\
\hline$h_{a_{\mathrm{FAT}}}^{2}$ & $0.24 \pm 0.09$ & 0.23 & $0.09 ; 0.45$ & $0.24 \pm 0.08$ & 0.24 & $0.10 ; 0.39$ & $0.20 \pm 0.07$ & 0.19 & $0.08 ; 0.35$ \\
\hline
\end{tabular}

PM, posterior mean; PD, posterior median; 95\% PPI, 95\% posterior probability interval; SD, posterior standard deviation.

in other studies (Johnson et al. 1993; Moser et al. 1998). For example, Johnson et al. (1993) reported an estimate of 0.14 in Brangus cattle. Moser et al. (1998) reported a low heritability estimate of 0.11 in the Brangus breed. These authors reasoned that the low heritability estimates they obtained for FAT were associated with the low phenotypic mean, probably because of age and level of nutrition (Seroba et al. 2011).

Results from this study showed that differences in posterior density between heavy-tailed distributions and Normal distribution are consistent with the find- ings of Kizilkaya et al. (2010) in their evaluation of bivariate Student's $t$ and Normal models for gestation length and birthweight for Piemontese cattle. It is important to note, however, that in contrast to results in this article, Cardoso et al. (2005) and Chang et al. (2006) found no real difference in PM estimates for heritabilities whether using Student's $t$, Slash or Normal models.

Posterior means of additive direct genetic correlations are in Table 7 . The posterior means of genetic correlations between HCW and REA and between HCW and FAT from MN, MSt and MS models were

Table 7 Posterior inference on genetic correlations between additive effects for hot carcass weight (HCW), longissimus muscle area (REA) and fat thickness (FAT) using multivariate Normal (MN), Student's $t$ (MSt) and Slash (MS) models

\begin{tabular}{|c|c|c|c|c|c|c|c|c|c|}
\hline \multirow[b]{2}{*}{ Genetic correlation } & \multicolumn{3}{|l|}{ MN } & \multicolumn{3}{|l|}{ MSt } & \multicolumn{3}{|l|}{ MS } \\
\hline & $\mathrm{PM} \pm \mathrm{SD}$ & PD & 95\%PPI & $\mathrm{PM} \pm \mathrm{SD}$ & PD & $95 \% \mathrm{PPI}$ & $\mathrm{PM} \pm \mathrm{SD}$ & PD & 95\%PPI \\
\hline$r_{\text {HCW, REA }}$ & $0.31 \pm 0.37$ & 0.31 & $-0.47 ; 0.92$ & $0.24 \pm 0.12$ & 0.25 & $-0.01 ; 0.44$ & $0.16 \pm 0.17$ & 0.18 & $-0.24 ; \quad 0.45$ \\
\hline$r_{\mathrm{HCW}, \mathrm{FAT}}$ & $-0.06 \pm 0.45$ & 0.02 & $-0.83 ; 0.67$ & $0.13 \pm 0.19$ & 0.15 & $-0.33 ; 0.42$ & $0.02 \pm 0.25$ & 0.05 & $-0.62 ; \quad 0.43$ \\
\hline$r_{\text {REA,FAT }}$ & $-0.38 \pm 0.27$ & -0.41 & $-0.78 ; 0.23$ & $-0.47 \pm 0.15$ & -0.46 & $-0.82 ;-0.20$ & $-0.57 \pm 0.17$ & -0.57 & $-0.88 ;-0.23$ \\
\hline
\end{tabular}

PM, posterior mean; PD, posterior median; 95\% PPI, 95\% posterior probability interval; SD, posterior standard deviation. 
found to be low, and 95\% PPI for these correlations from the three models used in this study included 0 indicating that correlations between these traits are non-significant. The posterior means of genetic correlations between HCW and REA from MN, MSt and MS models are lower than values reported in Riley et al. (2002) for Brahman breed, but are within the range (0.12-0.80) reported by Bertrand et al. (2001). The genetic correlation between HCW and FAT in this study is consistent with reports in the literature. The genetic correlation between REA and FAT showed a significant antagonistic relationship for MSt and MS models, which is consistent with results from literature (Riley et al. 2002; Seroba et al. 2011).

Multivariate residual distributions can be assumed Normal, Student's t or Slash. Model comparisons using DIC favoured MSt over MS and MN models, respectively. Smaller PM values of $v$ for MSt and MS models confirmed that the assumption of normally distributed residuals was inadequate for analysis of HCW, REA and FAT data sets. Our results support an antagonistic genetic relationship between REA and FAT.

\section{Acknowledgements}

The authors wish to acknowledge support from the Conference Attendance Fund from Berry College to present the results in this paper at the ADSA-ASAS Joint Meeting at Indianapolis, Indiana in July 2013. Financial support from the Scientific and Technological Research Council of Turkey (TUBITAK), provision of high-speed computer network by the Turkish Academic Network and Information Centre (ULAKBIM) and data collection and management assistance from Ellen J. Chaffee are gratefully acknowledged. Additional support provided by the College of Agriculture and Life Sciences, Cornell University, Ithaca, NY, Zoetis, Inc., National Research Initiative Competitive Grant Program (Grant No. 2006-35205-16864) from the USDA National Institute of Food and Agriculture; USDA-NIFA Research Agreements (Nos. 2009-6520505635, 2010-34444-20729) and USDA Federal formula Hatch funds appropriated to the Cornell University Agricultural Experiment Station are gratefully acknowledged. USDA is an equal opportunity provider and employer.

\section{References}

Albert J.H., Chibs S. (1993) Bayesian analysis of binary and polychotomous response data. J. Am. Stat. Assoc., 88, 669-679.
Bertrand J.K., Green R.D., Herring W.O., Moser D.W. (2001) Genetic evaluation for beef carcass traits. J. Anim. Sci., 79(E.Suppl.), E190-E200.

Bink M.C.A.M., Quaas R.L., Van Arendonk J.A.M. (1998) Bayesian estimation of dispersion parameters with a reduced animal model including polygenic and QTL effects. Genet. Sel. Evol., 30, 103-125.

Cardoso F.F., Rosa G.J.M., Tempelman R.J. (2005) Multiple-breed genetic inference using heavy-tailed structural models for heterogeneous residual variances. J. Anim. Sci., 83, 1766-1779.

Cardoso F.F., Rosa G.J.M., Tempelman R.J. (2007) Accounting for outliers and heteroskedasticity in multibreed genetic evaluations of postweaning gain of Nellore-Hereford cattle. J. Anim. Sci., 85, 909-918.

Chang Y.M., Andersen-Ranberg I.M., Heringstad B., Gianola D., Klemetsdal G. (2006) Bivariate analysis of number of services to conception and days open in Norwegian red using a censored threshold-linear model. J. Dairy Sci., 89, 772-778.

Chib S., Greenberg E. (1995) Understanding the Metropolis-Hastings algorithm. Am. Stat., 49, 327-335.

Crews D.H., Kemp R.A. (2001) Genetic parameters for ultrasound and carcass measures of yield and quality among replacement and slaughter beef. J. Anim. Sci., 79, 3008-3020.

Crews D.H., Kemp R.A. (2002) Genetic evaluation of carcass yield using ultrasound measures on young replacement beef cattle. J. Anim. Sci., 80, 1809-1818.

Crews D.H. Jr, Pollak E.J., Weaber R.L., Quaas R.L., Lipsey R.J. (2003) Genetic parameters for carcass traits and their live animal indicators in Simmental cattle. J. Anim. Sci., 81, 1427-1433.

Johnson M.Z., Schalles R.R., Dikeman M.E., Golden B.L. (1993) Genetic parameter estimates of ultrasound-measured longissimus muscle area and 12th rib fat thickness in Brangus cattle. J. Anim. Sci., 71, 2623-2630.

Kizilkaya K., Tempelman R.J. (2005) A general approach to mixed effects modeling of residual variances in generalized linear mixed models. Genet. Sel. Evol., 37, 31-36.

Kizilkaya K., Banks B.D., Carnier P., Albera A., Bittante G., Tempelman R.J. (2002) Bayesian inference strategies for the prediction of genetic merit using threshold models with an application to calving ease scores in Italian Piedmontese cattle. J. Anim. Breed. Genet., 119, 209-220.

Kizilkaya K., Carnier P., Albera A., Bittante G., Tempelman R.J. (2003) Cumulative t-link threshold models for the genetic analysis of calving ease scores. Genet. Sel. Evol., 35, 489-512.

Kizilkaya K., Garrick D.J., Fernando R.L., Mestav B., Yildiz M.A. (2010) Use of linear mixed models for genetic evaluation of gestation length and birth weight allowing for heavy-tailed residual effects. Genet. Sel. Evol., 42, 1-13.

MacNeil M.D., Nkrumah D.J., Woodward B.W., Northcutt S.L. (2010) Genetic evaluation of Angus cattle for carcass 
marbling using ultrasound and genomic indicators. $J$.

Anim. Sci., 88, 517-522.

Marshall D.M. (1994) Breed differences and genetic parameters for body composition traits in beef cattle. $J$. Anim. Sci., 72, 2745-2755.

Meyer K. (2007) Multivariate analyses of carcass traits for Angus cattle fitting reduced rank and factor analytic models. J. Anim. Breed. Genet., 124, 50-64.

Moser D.W., Bertrand J.K., Misztal I., Kriese L.A., Benyshek L.L. (1998) Genetic parameter estimates for carcass and yearling ultrasound measurements in Brangus cattle. J. Anim. Sci., 76, 2542-2548.

Nephawe K.A., Cundiff L.V., Dikeman M.E., Crouse I.D., Van Vleck L.D. (2004) Genetic relationships between sex-specific traits in beef cattle: Mature weight, weight adjusted for body condition score, height and body condition score of cows and carcass traits of their steer relatives. J. Anim. Sci., 82, 647-653.

Peters S.O., Kizilkaya K., Garrick D.J., Fernando R.L., Reecy J.M., Weaber R.L., Silver G.A., Thomas M.G. (2012) Bayesian genomewide association of growth and yearling ultrasound measures of carcass traits in Brangus heifers. J. Anim. Sci., 90, 3398-3409.

Peters S.O., Kizilkaya K., Garrick D.J., Fernando R.L., Pollak E.J., De Donato M., Hussain T., Imumorin I.G. (2013) Application of multivariate heavy-tailed distributions to residuals in the estimation of genetic parameters for growth traits in beef cattle. J. Anim. Sci., 91, 15521561.
Riley D.G., Chase C.C. Jr, Hammond A.C., West R.L., Johnson D.D., Olson T.A., Coleman S.W. (2002) Estimated genetic parameters for carcass traits of Brahman cattle. J. Anim. Sci., 80, 955-962.

Rosa G.J.M., Padovani C.R., Gianola D. (2003) Robust linear mixed models with normal independent distributions and Bayesian MCMC implementation. Biometrical J., 45, 573-590.

Seroba M.M., Maiwashe A., Nephawe K.A., Norris D. (2011) Genetic parameter estimates for live animal ultrasound measures of carcass traits in South African Angus cattle. S. Afr. J. Anim. Sci., 41, 1-7.

Sorensen D., Gianola D. (2002) Likelihood, Bayesian and MCMC Methods in Quantitative Genetics. Springer, New York, NY.

Sorensen D.A., Andersen S., Gianola D., Korsgaard I. (1995) Bayesian inference in threshold models using Gibbs sampling. Genet. Sel. Evol., 27, 229-249.

Spiegelhalter D.J., Best N.G., Carlin B.P., van der Linde A. (2002) Bayesian measures of model complexity and fit (with discussion). J R Stat Soc Series B, 64, 583-639.

Stranden I., Gianola D. (1999) Mixed effects linear models with t-distributions for quantitative genetic analysis: a Bayesian approach. Genet. Sel. Evol., 31, 25-42.

Utrera A.R., Van Vleck L.D. (2004) Heritability estimate for carcass traits of cattle: a review. Genet. Mol. Res., 3, 380-394. 